\title{
Performance of ATLAS Tracking Detector
}

\author{
V. Lacuesta* on behalf the ATLAS collaboration \\ IFIC (Universitat de Valencia - CSIC), Spain \\ E-mail: vicente.lacuesta@ific.uv.es
}

\begin{abstract}
The track and vertex reconstruction algorithms of the ATLAS Inner Detector have demonstrated excellent performance in the early data from the LHC. However, the rapidly increasing number of interactions per bunch crossing introduces new challenges both in computational aspects and physics performance. The combination of both silicon and gas based detectors provides high precision impact parameter and momentum measurement of charged particles, with high efficiency and small fake rate. Vertex reconstruction is used to identify the hard scattering process with high efficiency and to measure the amount of pile-up interactions, both aspects are crucial for many physics analyses. The performance of track and vertex reconstruction efficiency and resolution achieved in the 2011 and 2012 data-taking period are presented.
\end{abstract}

VERTEX 2012

Jeju, Korea

September, 16-21 2012

\footnotetext{
* Speaker.
} 


\section{Contents}

\section{Introduction}

The Large Hadron Collider [?] is a particle physics accelerator built at CERN. It collides headon bunches of protons or heavy ions. The analysis of these collisions have lead to the discovery of a new Higgs Boson like particle. The ATLAS experiment [?] is a general purpose experiment that records collision events produced by the LHC. In order to fulfil its scientific objectives, ATLAS is equipped with an Inner Detector (ID) tracking system [?] embedded in a $2 \mathrm{~T}$ axial magnetic field surrounding the interaction point. The calorimetry system [?, ?] is located beyond the ID solenoid and combines two techniques: liquid argon and sampling technique with plastic scintillators embedded in an iron absorber. Finally a muon detector system [?] occupies the outermost region of the detector and uses an air-core toroid with strong bending power in a large volume within a light and open structure.

The LHC started its operations late 2009, by colliding proton beams at $900 \mathrm{GeV}$ center of mass energy. During 2010 and 2011 the LHC produced proton-proton collisions at $7 \mathrm{TeV}$. The integrated luminosity recorded by ATLAS amounts to $40 \mathrm{pb}^{-1}$ in 2010 and $5.25 \mathrm{fb}^{-1}$ in 2011. During the year 2012 the LHC is operating at $8 \mathrm{TeV}$ and more than $12 \mathrm{fb}^{-1}$ of data has been recorded until now. LHC also collided heavy ions (lead) in 2010 and 2011.

This note summarizes the tracking and vertexing performance of the ATLAS ID and highlights the studies on tracking and vertex recontruction, and detector alignment.

\section{The ATLAS Inner Detector}

The ATLAS ID acts as the tracking system for charged particles. It was designed to produce a robust and efficient track reconstruction. It consists of three subdetectors: two using silicon planar technology (pixels and microstrip) and a drift chamber using straw tubes filled with gas. The subdetectors are: Pixel, SCT and TRT respectively. All the three subsystems are divided in three parts: a barrel and two end-caps (figure ??). The barrel part is composed of several cylindrical layers of modules. Each end-cap consists of several disks or wheels of modules.

The superconducting solenoid which surrounds the entire tracking system produces a $2 \mathrm{~T}$ axial magnetic field along the beam axis direction. This magnetic field bends the trajectory of the charged particles and allows the measurement of their momentum. The Pixel detector is the innermost of the ATLAS subsystems. The 1744 silicon pixel modules [?] are arranged in 3 concentric barrel layers and 3 disks in each of the end-caps. Each module has an active area of $16.4 \times 60.8 \mathrm{~mm}^{2}$ and it is segmented in pixels of $50 \times 400 \mu \mathrm{m}^{2}$. The first barrel layer is located at just $5 \mathrm{~cm}$ from the beam axis. The silicon microstrip tracker (SCT) is built from modules assembled with two 
pairs of $6 \mathrm{~cm}$ long wafers with a pitch of $80 \mu \mathrm{m}$ [?, ?]. Each side of the module of $12 \mathrm{~cm}$ is composed by two chained wafers. Each pair of sensors is glued back to back with a $40 \mathrm{mrad}$ stereo angle. There are in total 4088 SCT modules distributed in 4 concentric barrel layers and 9 disks in each of the two end-cap structures. The four wafers of each SCT modules were assembled under strict tolerances. The mechanical tolerance for positioning sensors within the back-to-back pair was required to be better than $5 \mu \mathrm{m}$ transverse to the strip direction. Thanks to that, the SCT module is considered as the basic alignable unit. The Transition Radiation Tracker (TRT) [?, ?] is the outermost of the ID subdetectors (its outer radius is just above 1 meter). The detector consists of 298304 straw tubes $4 \mathrm{~mm}$ diameter. Each straw has a sensing wire in the center. The straws are arranged in 96 barrel modules (staggered in 3 rings) and 40 wheels in each end-cap. Besides the tracking capabilities, the TRT may identify electrons thanks to the transition radiation. The TRT use a gas mixture of $\mathrm{Xe}, \mathrm{CO}_{2}$ and $\mathrm{O}_{2}$. Table ?? presents a summary of the main characteristics of the three ID subdetectors,including its nominal resolution and number of readout channels.

\begin{tabular}{lccccc}
\hline \hline Subdetector & $\begin{array}{c}\text { Radius } \\
{[\mathrm{cm}]}\end{array}$ & Element size & $\begin{array}{c}\text { Resolution } \\
\text { in precision coordinate }\end{array}$ & $\begin{array}{c}\text { Hits/track } \\
\text { in the barrel }\end{array}$ & $\begin{array}{c}\text { Readout } \\
\text { channels }\end{array}$ \\
\hline Pixel & $5-12$ & $50 \mu \mathrm{m} \times 400 \mu \mathrm{m}$ & $10 \mu \mathrm{m} \times 115 \mu \mathrm{m}$ & 3 & $80 \times 10^{6}$ \\
SCT & $30-52$ & $80 \mu \mathrm{m}$ & $17 \mu \mathrm{m}$ & 8 & $6 \times 10^{6}$ \\
TRT & $56-107$ & $4 \mathrm{~mm}$ & $130 \mu \mathrm{m}$ & 30 & $3.5 \times 10^{5}$ \\
\hline \hline
\end{tabular}

Table 1: Summary of the main characteristics of the three ATLAS ID subdetectors. The resolution is measured transversal to the strips in the SCT case and radial distance to the straw wires in the TRT case.

\section{Track reconstruction}

Tracks are reconstructed in the inner detector using a sequence of algorithms [?]. The insideout algorithm starts from 3-point seeds in the silicon detectors and adds hits using a combinatorial Kalman filter. Ambiguities in the track candidates found in the silicon detectors are resolved, and tracks are extended into the TRT. The inside-out algorithm is designed for the efiñ Acient reconstruction of charged particles emerging from the interaction point. The tracks reconstructed
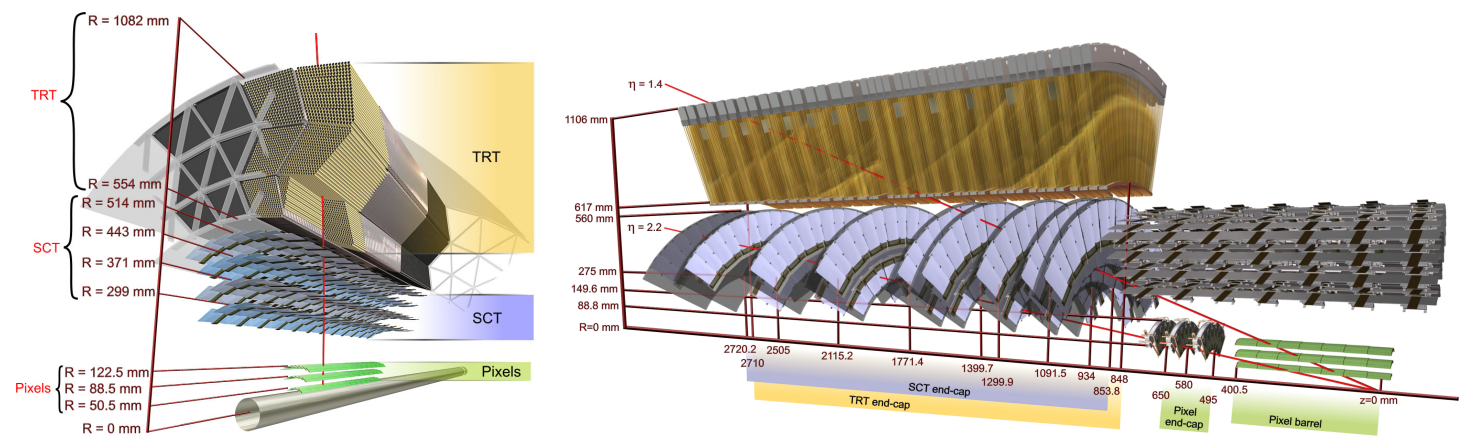

Figure 1: Schematic view of the layout of the ID barrel (left) and end-caps (right). 
by the inside-out algorithm are required to have transverse momentum $p_{T}>400 \mathrm{MeV}$. In a second stage, a track search starts from segments reconstructed in the TRT and extends them inwards by adding silicon hits, which is referred to as back-tracking. Back-tracking is designed to reconstruct secondaries, which are particles produced in the interactions of primaries. Finally tracks with a TRT segment but no extension into the silicon detectors are referred to as TRT-standalone tracks. The track reconstruction efficiency is defined as the fraction of primary particles with $p_{T}>400$ $\mathrm{MeV}$ and pseudorapidity $(|\eta|)<2.5$ matched to a reconstructed track. In the figure ?? the track reconstruction efficiency as a function of $p_{T}$ and $\eta$ between samples containing no pile-up $(\mu=1)$ and samples containing significant pile-up is compared.
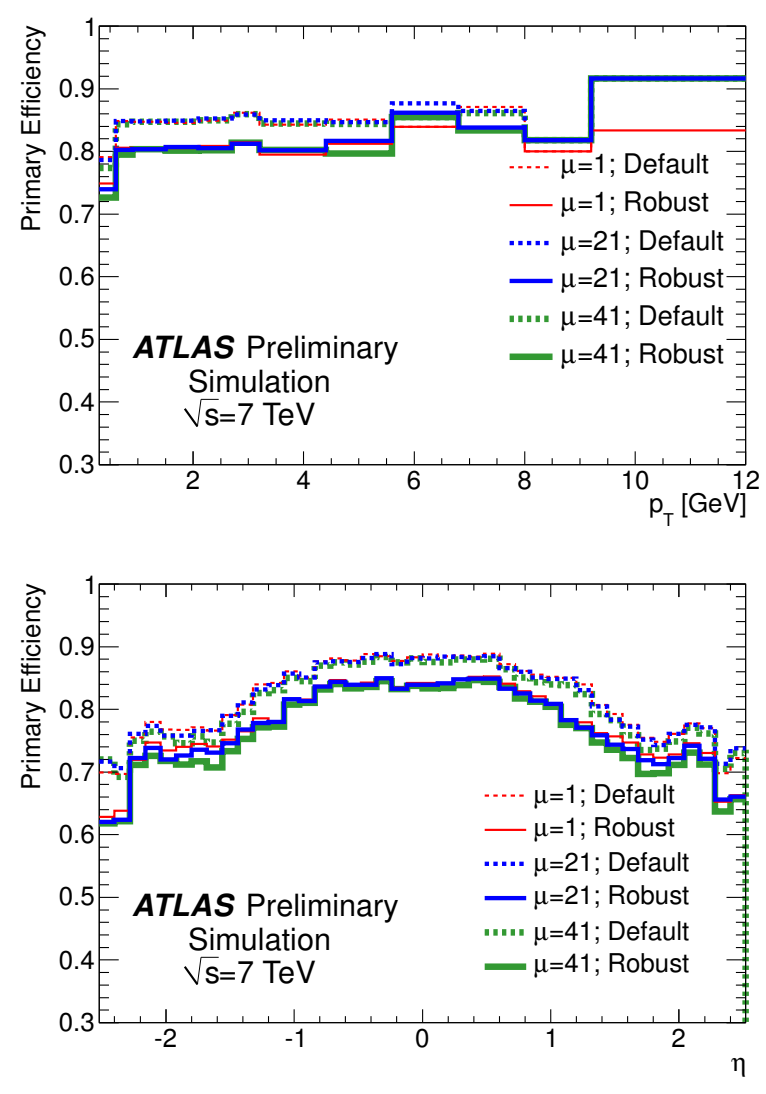

Figure 2: The primary track reconstruction efficiency in minimum bias Monte Carlo samples containing exactly one and on average 21 or 41 interactions. The distributions are shown for tracks passing the default (dashed) and robust (solid) requirements [?].

\section{Vertex reconstruction}

Primary vertices are reconstructed using an iterative vertex finding algorithm [?]. Vertex seeds are obtained from the z-position at the beamline of the reconstructed tracks. An iterative $\chi^{2}$ fit is made using the seed and nearby tracks. Each track carries a weight which is a measure of its compatibility with the fitted vertex depending on the $\chi^{2}$ of the fit. Tracks displaced by more than 
$7 \sigma$ from the vertex are used to seed a new vertex and the procedure is repeated until no additional vertices can be found. The beam spot position is used as a three-dimensional constraint. During reconstruction vertices are required to contain at least two tracks.

The efficiency to reconstruct a vertex from a minimum bias interaction is shown in Figure ??(a) as a function of the average number of interactions per bunch crossing. This is shown for vertices reconstructed from tracks passing the default requirements and for those reconstructed from tracks passing the robust requirements. The default requirements are at least 7 silicon hits and at most 2 holes (missing hits) in the Pixel detector. The robust selection requires 9 silicon hits and no holes. The primary vertex resolution depends on the number of reconstructed charged particles and their pt. In the transverse plane the estimated resolution is $23 \mu \mathrm{m}$. The vertex efficiency is calculated with the same track-to-particle matching used to calculate the tracking efficiency. Vertices are matched to interactions by calculating the sum of the weights of the tracks in a vertex matched to each interaction. The increasing number of fake tracks in a high pile-up environment increases the probability to reconstruct a fake vertex. In simulation, a vertex is defined as fake if the leading contribution to the total weight of the vertex is from fake tracks. The fraction of fake vertices reconstructed is shown in Figure ??(b) for two different selections.
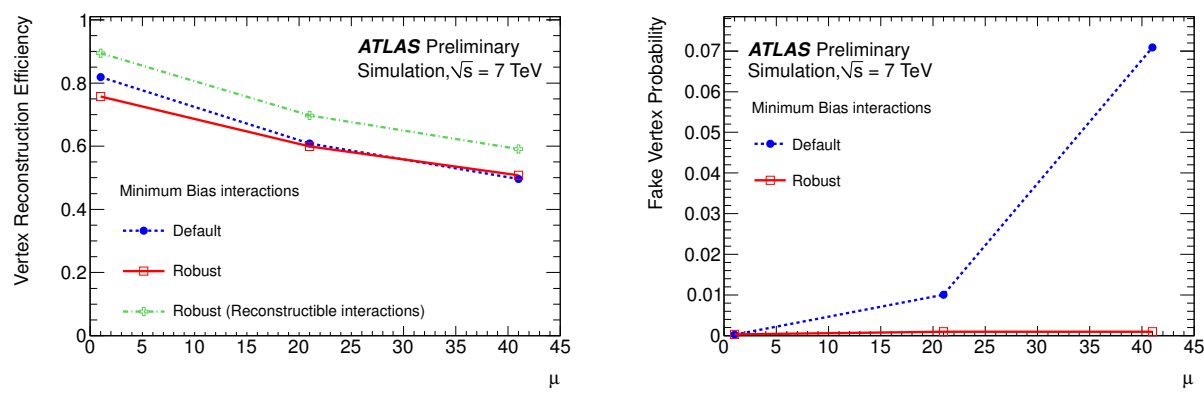

Figure 3: The vertex reconstruction efficiency (left) and fake probability (right) as a function of the average number of interactions in minimum bias Monte Carlo simulation. These are shown both using default track selection (blue, dashed) and with the robust track requirements (red, solid). The vertex reconstruction efficiency with the robust track requirements is shown for reconstructible interactions (green, dot-dashed), defined as having at least two stable charged primary particles with $|\eta|<2.5$ and $p_{T}>400$ [?]

\section{Inner Detector Alignment}

The goal of the alignment is to provide an accurate description of the geometry of the detector: the location and orientation of every tracking element. This is vital for a proper and precise determination of the track parameters and to avoid biases. The ID has been aligned using a track based method [?]. The algorithm is based on the minimisation of a $\chi^{2}$ constructed from track to hit residuals with respect to both the alignment and track parameters $[?, ?]$. The alignment procedure is executed at 3 different levels in accordance with the assembly of the ID structures. The level 1 corresponds to the large barrel and end-cap structures of the Pixel, SCT and TRT (just 7 structures to align). The level 2 deals with the barrel layers and end-cap disks of the Pixel and SCT (31 structures) and the barrel modules and end-cap wheels of the TRT (176 structures). Finally, the 

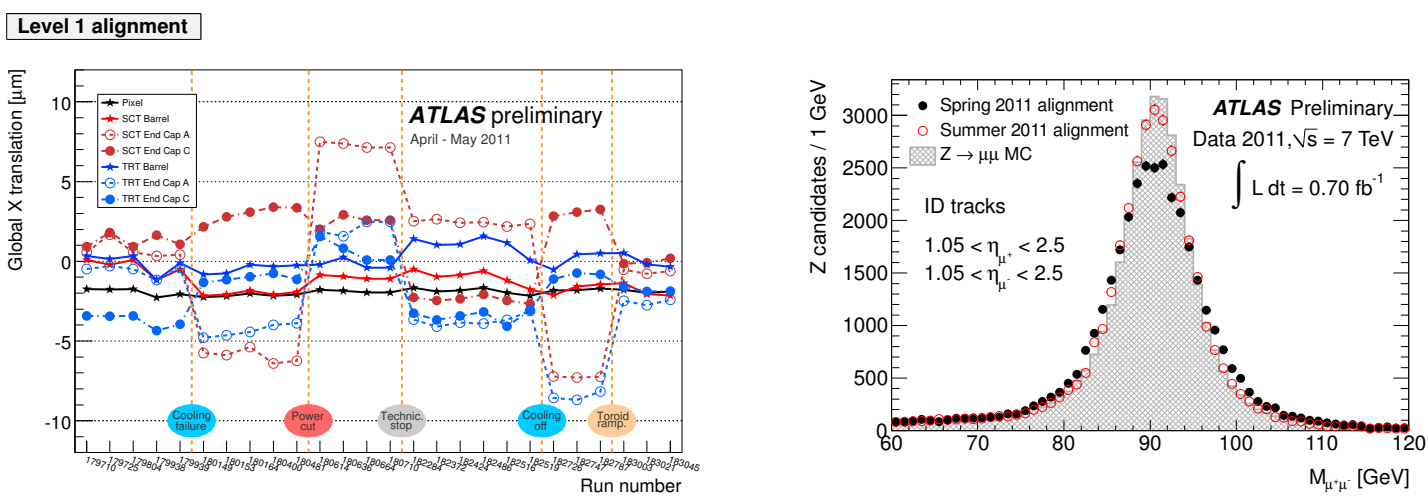

Figure 4: Left: Evolution of the corrections to the to the position of the each sub-detector. The data spans over a period of 2 month. The detector only moves when reacting to hardware events. Otherwise, it is remarkably stable. Right: Reconstructed invariant mass of the $Z \rightarrow \mu^{+} \mu^{-}$in the Endcap A. The improvement of the summer 2011 alignment is clearly seen. MC with a perfectly aligned detector is also included [?].

level 3 aligns each Pixel and SCT module and all TRT straw tubes. In total, one has to deal with more than 700k degrees of freedom. There are two dedicated data streams selected by the high level trigger: a collection of high $p_{T}$ and isolated collision tracks and a set of cosmic-ray tracks triggered during the empty LHC bunches. The detector stability is tested run-by-run (figure ?? left). Through the alignment algorithm it is possible to set constraints on the track and alignment parameters: contstrain the tracks to come from the beam spot region, the momentum $(p)$ of muons from the Muon Spectrometer, the energy over momentum $(E / p)$ for electrons and assembly survey data [?], etc. The reached alignment precision of the pixel barrel modules is $4 \mu \mathrm{m}$ and $10 \mu \mathrm{m}$ for the SCT modules. A nice data vs. MC agreement is obtained for the residuals of all parts of the ID [?]. The alignment validation comprises a detailed check of many alignment specific distributions (as the residuals of all components) as well as checks for track parameters and their errors. The resonance invariant masses (light as $K_{s}^{0}$ and heavy as $Z$, figure ?? right) are scrutinized against all the track parameters in order to detect and correct possible biases.

\section{Summary and Outlook}

The ATLAS Inner Detector tracking system performance has been discussed. The Pixel, SCT and TRT are operating very efficiently and are providing high quality data for the physics analysis teams. On the offline front, all the efforts dedicated to study the precise positioning of the hits recorded by the sensors, to understand the material inside the tracking volume, pattern recognition and track fitting, primary and secondary vertex fit, detector alignment and particle identification already bear fruit as the ID performance is rapidly reaching its design level. The performance of the ID tracking has allowed an efficient tracking during the LHC 2010 heavy ion run, which in its turn allowed to prepare the tracking for the larger interaction pile-up in 2011 and 2012 and also for the high luminosity LHC in the next years. 


\section{References}

[1] L. Evans and P. Bryant, LHC Machine, J. Instrum. 3 (2009) S08001.

[2] ATLAS Collaboration, The ATLAS experiment at the CERN Large Hadron Collider, J. Instrum. 3 (2009) S08003.

[3] ATLAS Collaboration, Alignment requirements, in ATLAS Inner Detector Technical Design Report, vol. I, p. 215. CERN-LHCC-1997-016, CERN, Geneva, 1997.

[4] ATLAS Collaboration, Readiness of the ATLAS liquid argon calorimeter for LHC collisions, Eur. Phys. J. C70 (2010) 723-753, DOI : $10.1140 /$ p jc/s10052-010-1354-y.

[5] ATLAS Collaboration, Readiness of the ATLAS Tile Calorimeter for LHC collisions, Eur. Phys. J. C70 (2010) 1193-1236, DOI: $10.1140 / \mathrm{epjc} / \mathrm{s} 10052-010-1508-y$.

[6] ATLAS Collaboration, Commissioning of the ATLAS Muon Spectrometer with cosmic rays, Eur. Phys. J. C70 (2010) 875-916, DOI : $10.1140 /$ ep jc/s 10052-010-1415-2.

[7] G. Aad et al., ATLAS pixel detector electronics and sensors, J. Instrum. 3 (2008) P09004.

[8] A. Abdesselam et al., The barrel modules of the ATLAS semiconductor tracker, Nucl. Inst. Meth. A 568 (2006) 642-671.

[9] A. Abdesselam et al., The ATLAS semiconductor tracker end-cap module, Nucl. Inst. Meth. A 575 (2007) 98-118.

[10] E. Abat et al., The ATLAS TRT Barrel Detector, J. Instrum. 3 (2008) P02014.

[11] E. Abat et al., The ATLAS TRT end-cap detectors, J. Instrum. 3 (2008) P10003.

[12] ATLAS Collaboration Performance of the ATLAS Inner Detector Track and Vertex Reconstruction in the High Pile-Up LHC Environment, ATLAS-CONF-2012-042.

[13] ATLAS Collaboration, Alignment of the ATLAS Inner Detector Tracking System with 2010 LHC proton-proton collisions at $\sqrt{s}=7 \mathrm{TeV}$, ATLAS-CONF-2011-012.

[14] P. Bruckman, A. Hicheur and S. Haywood, Global $\chi^{2}$ approach to the alignment of the ATLAS silicon tracking detectors, ATLAS Note ATL-INDET-PUB-2005-002.

[15] A. Bocci and W. Hulsbergen, TRT alignment for SR1 cosmics and beyond, ATL-INDET-PUB-2007-009.

[16] A. Andreazza, V. Kostyukhin and R.J. Madras, Survey of the ATLAS pixel detector components, ATLAS Note ATL-INDET-PUB-2008-012.

[17] https://twiki.cern.ch/twiki/bin/view/AtlasPublic/InDetTrackingPerformanceApprovedPlots. 